\title{
The Influence of Different Important Map for Image Retargeting
}

\author{
Zijuan Zhang \\ Shaanxi Polytechnic Institute \\ Xianyang, 712000, China
}

\begin{abstract}
Under the condition of limited resources of the display, image resizing has become a hot issue in the field of image processing. Image retargeting algorithm can satisfy these conditions simultaneously: protecting the important content of the source information; avoiding the important content of excessive deleting; making full use of resources in adaptation results to keep a good sense. The first stage of image retargeting algorithm is to extract important map of the source information, important map reflects the human eye vision for source information degree of different content of interest. Importance map's accuracy directly affects the final resizing result. In the vast majority of cases, under the condition of the same kind of resizing method, important map's accuracy is more precise, the retargeting effect is better. Therefore, research emphasis on retargeting algorithm can not only focus on adaptation stage, perception stage should also be adequately highlighted.
\end{abstract}

Keywords-image resizing; image retargeting; important map; influence

\section{INTRODUCTION}

With the diversification of multimedia display devices, the sizes of these devices range from high resolution PC to low resolution smart phone, then the image must be resized to fit the screen of these devices. Under the condition of the limited resources of display source information, image retargeting has become a hot issue in the field of image processing.

Avidan etc. [1] has proposed the "Content perception" concept on the Siggraph 2007, and the corresponding image retargeting algorithm has been given. The algorithm firstly calculates important map of the source image by gradient model, importance map reflects the source image's important degree of the different content, which represents the interested degree of human eye about all kinds of content; Then according to the important map using difference operation, making the change during the process of adaptation in the unimportant areas, so as to further protect the important content of the source image, realizing the content aware. This algorithms such as Avidan's was called Seam Carving method(Backward Seam Carving, BSC)[1]. As the BSC algorithm is put forward, on the issue of the image retargeting, international scholars and research institutions proposed many adaptation method, and built a test database [2-4] and quality evaluation model. To realize image retargeting is a challenging problem, because the adaptation algorithm needs to meet these conditions at the same time: protecting the important content of the source information, avoiding the important content's excessive deleting, making full use of resources in adaptation results. Firstly, the algorithm usually extracts the important map to represent the content's importance of each pixel in the original information, then according to the important map and may consider additional constraints, retargeting method is applied to adjust the size of the source information.

\section{ALGORITHM FRAMEWORK}

Image retargeting algorithm is mainly divided into stages of perception (first phase) and the adapter (second phase). Figure 1 shows the basic framework of the algorithm. Among them, the perceptual stage to extract the important map of the source information, as shown in figure 1(a). To achieve perception adaptation, in addition to the perceptual stage to extract important map, also needing adaptation method based on important degree in the adaptation phase, as shown in figure 1(b).

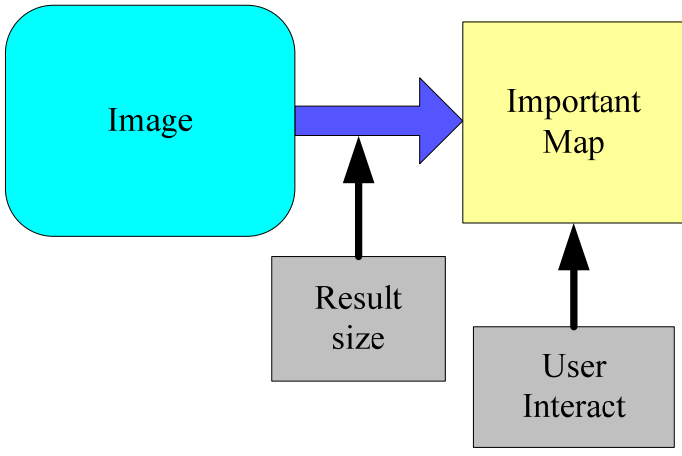

(a) perceptual stage

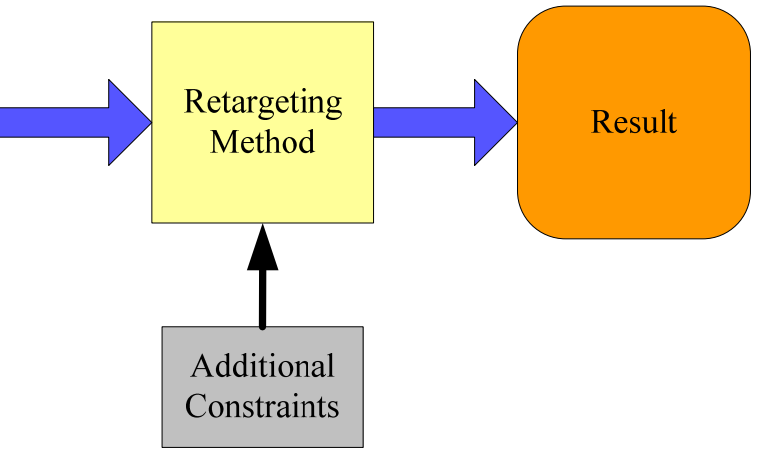

(b) adaptation stage

FIGURE I. THE ALGORITHM FRAMEWORK OF IMAGE RETARGETING 


\section{THE INFLUENCE OF DIFFERENT IMPORTANT MAP FOR IMAGE RETARGETING}

The first stage of retargeting algorithm is to extract the source information's important map, important degree in image reflects the human eye's vision interest for source information about different content. Automatically extracting the source information's important map is one of the important subjects about image retargeting research, many scholars do research on the issue. Among the existing algorithm, these methods can be divided into the following three categories:

\section{A. Gradient}

Gradient method is that the gradient operator is adopted for source information to calculate the source information's the gradient map[1]. The pixels with higher gradient value is thought to be more important; On the contrary, the pixels with lower gradient value is regarded as unimportant content. This method has the advantage with simple calculation, high speed, and can show the image's structure information. Deficiency is that the edge area is able to be identified and important content's internal information was mistakenly thought as unimportant content. As shown in figure 2 (b), using the gradient method as the important map of source information (figure 2) (a), gradient map only identify the outline of the source information, at the same time wrongly emphasis on the edge of the background lanterns.

\section{B. Saliency}

Saliency degree method is the application of any saliency degree model to extract the source information's important map. Exist retargeting algorithm with the saliency map commonly uses these models: Itti model[5], Hou model [6], Harel model [7], Goferman model[8], etc. The saliency map can identify the subject of the source information accurately, but not response structure's information well. Figure 2 (c) is extracted by Itti model, the important image accurately emphasizes the people in the source information, but not reflect the characteristics of the structure.

\section{Combination}

In order to absorb the advantages of the two kinds methods mentioned above, and overcome its shortcomings, we can use the combination of gradient map and saliency map as important map. Joint map can reflect the important content in the source information, its precision is much higher. Figure 2 (d) is the combined map of multiplying the gradient information (figure 2 (b)) and a saliency information (figure 2 (c)), it can be seen that the combined map has better effect.

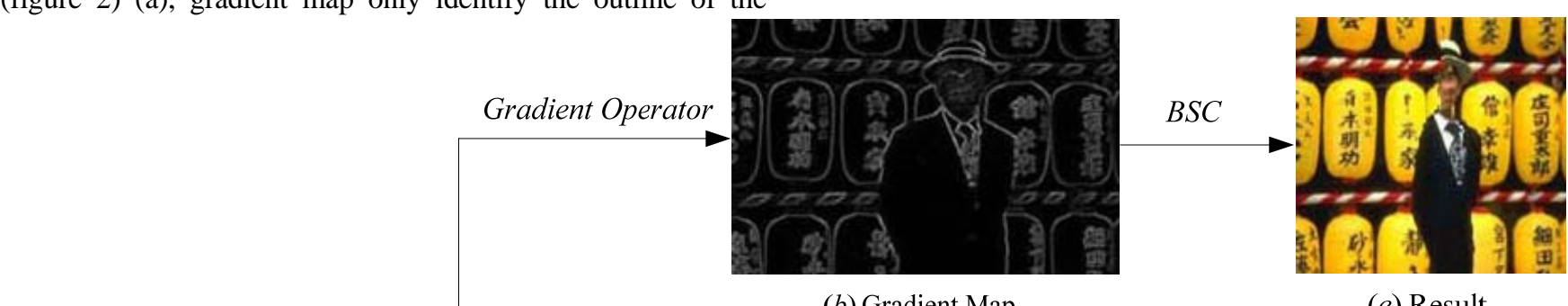

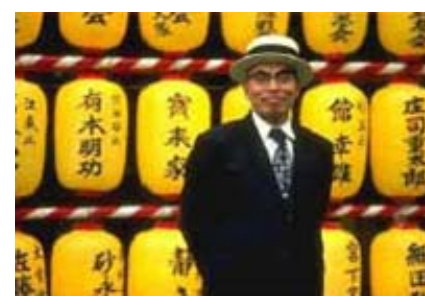

(a) Image (b) Gradient Map

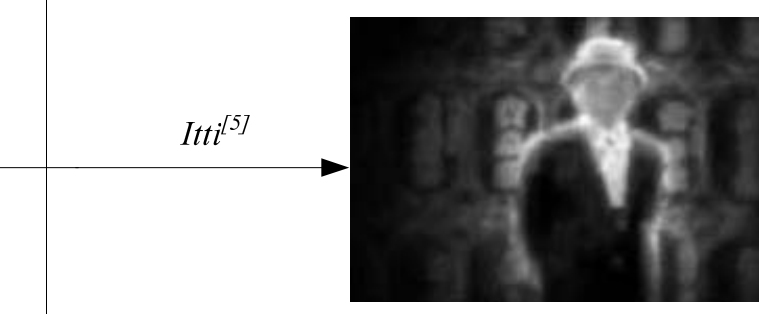

(c) Saliency Map

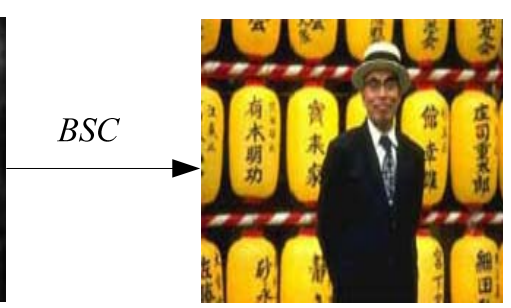

(f) Result

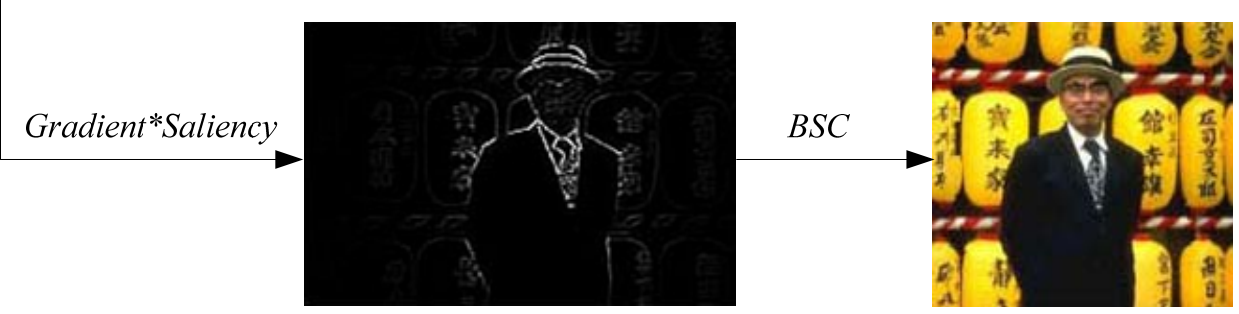

(d) Combination Map 


\section{CONCLUSION}

Importance map's accuracy directly affects the result of image retargeting. In the vast majority of cases, under the same kind of retargeting method, the final effect of retargeting is better with more accurate. We can obviously see that figure 2 (g) has the best resizing result, which not only maintains the subject of source information, but also achieves good integral effect, the reason is that the adaptation process is based on more accurate important map. Therefore, research on retargeting algorithm can not only focus on adaptation stage, perception stage should also be adequately highlighted.

\section{REFERENCES}

[1] S. Avidan, A. Shamir, "Seam carving for content-aware image resizing," ACM Transactions on Graphics, vol. 26(3), pp.1-10, 2007.
[2] http://people.csail.mit.edu/mrub/retargetme/[DB/OL]

[3] M. Rubinstein, A. Shamir, and S. Avidan, "Multi-operator media retargeting,” ACM Transactions on Graphics, vol. 28(3), pp.23, 2009.

[4] D. Simakov, Y. Caspi, and E. Shechtman, "Summarizing visual data using bidirectional similarity,” Proceedings of the IEEE Conference on CVPR, pp.1-8, 2008.

[5] L. Itti, C. Koch, and E. Ernst, "A model of saliency-based visual attention for rapid scene analysis," IEEE Transactions on Pattern Analysis and Machine Intelligence, vol. 20(11), pp.1254-1259, 1998.

[6] X.D. Hou, L.Q. Zhang, "Dynamic visual attention: searching for coding length increments,” Proceedings of the $22^{\text {nd }}$ Annual Conference on NIPS, pp, 681-688, 2008.

[7] J. Harel, C. Koch, and P.Perona, “Graph-based visual saliency," $20^{\text {th }}$ Annual Conference on Neural Information Processing Systems, pp.545552, 2007.

[8] S. Goferman, M.L. Zelnik, and A. Tal, "Context-aware saliency detection," IEEE Transactions on Pattern Analysis and Machine Intelligence, vol. 34(10), pp.2376-2383, 2011. 